\title{
Equidad gerencial, como una demanda intangible de mercado: hacia organizaciones sustentables ${ }^{1}$
}

\section{Equity management, as an intangible market demand: towards sustainable organizations}

\author{
K. Terán, J.M. Robles, J. M. Preciado, y D. C. López.
}

Recibido: agosto 26 de 2019 - Aceptado: octubre 30 de 2019.

\begin{abstract}
Resumen - El presente artículo da cuenta de los fundamentos referenciales de cómo el mercado condiciona a las organizaciones para competir con atributos intangibles del producto, mismos que se relacionan con los componentes básicos de la sustentabilidad (social, ambiental y económico). De igual manera fundamenta cómo la equidad gerencial, como una demanda intangible de mercado, propicia cambios organizacionales hacia la sustentabilidad. Estas bases conceptuales no aparecen de manera tácita en las revisiones de literatura, y constituyen una importante estructura conceptual para abordar el análisis de las organizaciones. En la literatura, las investigaciones que tratan el tema de la equidad gerencial, como una demanda intangible de mercado que propicia cambios organizacionales para transitar hacia la sustentabilidad, es limitada, y es crucial mencionar que el proceso de su integración en la gerencia se traduce en garantía de calidad y excelencia empresarial que da respuesta a una demanda intangible de mercado, la cual coadyuva a la conformación de organizaciones sustentables.
\end{abstract}

Palabras clave-Demanda intangible de mercado, equidad gerencial, organización sustentable, sustentabilidad.

${ }^{1}$ Producto derivado del proyecto de investigación "Equidad gerencial, como una demanda intangible de mercado: hacia organizaciones sustentables. El caso de uva de mesa". Presentado por el Grupo de Investigación del Centro de Investigación en Alimentación y Desarrollo (CIAD) en Hermosillo, Sonora.

K. Terán, Centro de Investigación en Alimentación y Desarrollo (CIAD), Hermosillo, Sonora, email: Karla.Terandr18@estudiantes.ciad.mx

J. M. Robles, Centro de Investigación en Alimentación y Desarrollo (CIAD), Hermosillo, Sonora, email: jrobles@ciad.mx

J. M. Preciado, Centro de Investigación en Alimentación y Desarrollo (CIAD), Hermosillo, Sonora, email: mpreciado@ciad.mx

D. C. López, Universidad Católica de Pereira, Pereira, Colombia, email: dianac.lopez@ucp.edu.co

Como citar este artículo: Terán, K., Robles, J. M., Preciado, J. M., y López, D. C. Equidad gerencial, como una demanda intangible de mercado: hacia organizaciones sustentables, Entre Ciencia e Ingeniería, vol. 13, no. 26, pp. 85 93, julio-diciembre 2019. DOI: https://doi.org/10.31908/19098367.1166.

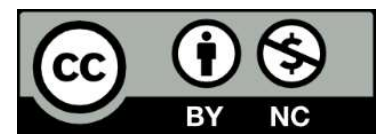

Attribution-NonCommercial 4.0 Intenational (CC By-NC 4.0)
Abstract - This article gives an account of the referential foundations of how the market conditions organizations to compete with intangible attributes of the product, which are related to the basic components of sustainability (social, environmental and economic). Likewise, the referential foundations of how equity management, as intangible market demand, propitiates organizational changes towards sustainability are provided. These conceptual bases do not appear tacitly in literature reviews and constitute an important conceptual structure to address the analysis of organizations. In the literature, research that deals with the issue of equity management, as an intangible market demand that encourages organizational changes to move towards sustainability, is limited, and it is crucial to mention that the process of its integration into management translates into guarantee of quality and business excellence that responds to an intangible market demand, which contributes to the creation of sustainable organizations.

Keywords-Equity management, intangible market demand, sustainable organization, sustainability.

\section{INTRODUCCIÓN}

$\mathrm{E}^{\mathrm{L}}$ presente artículo pretende indagar en la literatura - referentes teóricos ligados a las demandas intangibles de mercado, y cómo la equidad gerencial, siendo parte de esas exigencias que realizan los consumidores a las organizaciones, propicia cambios organizacionales hacia la sustentabilidad. Se busca con ello, presentar una profunda revisión del estado del arte que permita dar pertinencia a dicha conjetura como marco interpretativo para las nuevas problemáticas de las organizaciones.

Las demandas intangibles de mercado son aquellos requerimientos que realizan los clientes a la organización, que están relacionados con prácticas sustentables en los tres pilares del desarrollo sustentable (social, ambiental y económico). La equidad gerencial ha venido a sumarse a este grupo de demandas con el propósito de responder a la necesidad de incluir en los niveles gerenciales de la organización, a personas que cuenten con méritos, habilidades y competencias, independientemente de su sexo, ascendencia, religión, edad, entre otras. 
El término "equidad gerencial" es relativamente nuevo, pues éste ha sido abordado aproximadamente desde los años 90 , y ha sido aplicado en diversos campos y para distintos propósitos investigativos. En el año 2001, Dorsch y colaboradores examinaron más de cerca la equidad gerencial de los clientes, en donde el capital de los consumidores proporciona una nueva perspectiva estratégica para las decisiones de administración de ventas al permitir a los gerentes una base para identificar a los clientes o tipos de clientes; es decir, los que desean buscar, retener, evitar o incluso abandonar. Estos autores afirman que el éxito de una compañía dependerá en gran medida, de la habilidad del gerente para gestionar con efectividad todo el entorno del cliente, por lo que el declive continuo en la satisfacción de éste resultará de la falla en la respuesta a las expectativas de dicho cliente [1].

Para Bruhn, y coautores, la equidad gerencial del cliente abarca todas aquellas actividades que pretenden maximizar la equidad del consumidor; este enfoque al cliente desafía las prácticas empresariales tradicionales que a menudo se concentran en servicios y productos, en lugar de estar dirigidas a las relaciones con el cliente, que es lo que permitirá definir cuáles serán los objetivos y las estrategias de comercialización [2].

Cepeda y colaboradores por su parte coinciden en que la equidad gerencial como demanda del cliente, compromete a los gerentes de una firma a realizar esfuerzos específicos para adquirir, retener y desarrollar las relaciones con los clientes con el fin de maximizar el capital que estos invierten en la compañía; pero además, se busca su lealtad y compromiso a largo plazo. Dicha investigación proporciona una metodología para evaluar y agrupar clientes, en particular para determinar cuándo, cuánto y cómo invertir en ellos de acuerdo con su valor actual y potencial, así como a los ingresos económicos que ellos permiten en la compañía. En este sentido, los investigadores demuestran que el representante de la firma debe ordenar la cartera de sus clientes de acuerdo con la permanencia que pueda tener cada uno de ellos en la compañía [3].

Hwang y coautores, afirman que la equidad gerencial del cliente es fundamental para el éxito a largo plazo de una empresa, y esto se logra reteniendo clientes existentes o atrayendo nuevos prospectos. En su propuesta, hacen mención de un programa de lealtad que a largo plazo recompensa las compras repetitivas de los clientes para mejorar su lealtad y aumentar la rentabilidad de las empresas. Este estudio demuestra que existen medios de contacto que representan una alternativa para cuando el consumidor se encuentra físicamente lejos de la compañía, ya que juegan un papel regulador al aminorar los efectos demográficos y en definitiva, ofrecen un beneficio con la implementación de los programas de lealtad. Estos hallazgos permiten a la firma predecir la situación de sus clientes y favorecerlos, según sea el caso [4].

Como se puede apreciar, existen investigaciones en donde ya se ha incorporado el concepto de "equidad gerencial", pero solo hay una que lo relaciona con la equidad en los altos niveles jerárquicos de una organización, firma o institución; tal es el caso de Yates, quien afirma que la equidad gerencial es necesaria para aquellos que han sido históricamente poco representados, como por ejemplo grupos étnicos, gremios de diferentes razas, mujeres, inmigrantes, personas mayores que aún se encuentran en el mercado laboral, entre otros; y es ese enfoque de la equidad gerencial que permite que una organización se concentre en lo más importante: el trato a las personas y el uso de sus talentos y capacidades de la mejor manera posible [5].

Yates discute cómo la equidad gerencial fue introducida en los altos niveles jerárquicos de la Armada de Estados Unidos, y la manera en que sus estructuras han mejorado. De igual manera, el autor argumenta que, a través de la incorporación de este principio de equidad gerencial, Xerox, Kellogg, Avon, Chrysler y Johnson and Johnson, han sido casos de éxito. Estas organizaciones han acogido planes muy particulares para mejorar la utilización de su fuerza de trabajo, así como la calidad de su servicio y/o producto, colocándose en un lugar privilegiado en el mercado [5].

Es así como la equidad gerencial ha sido estudiada con el objeto de garantizar la "no discriminación empresarial" y el aprovechamiento de las capacidades del individuo en el puesto para de esta manera, generar nuevos modelos organizacionales y gerenciales. Sin embargo, se encontró solamente que un autor ha abordado dichos conceptos desde la perspectiva de la transformación organizacional o institucional. Cabe aclarar que son limitadas las investigaciones que contemplan a la equidad gerencial como una demanda intangible de mercado, y que la colocan como un medio para propiciar cambios en las organizaciones que las llevan a transitar hacia la sustentabilidad. Es en esta línea, donde se ubica la originalidad y aportación del presente artículo.

\section{II.DEMANDAS INTANGIBLES DE MERCADO: EQUIDAD GERENCIAL}

\section{A. Demandas intangibles de mercado}

Para abordar el concepto de demandas intangibles de mercado, es pertinente discutir primero el concepto básico de "mercado", y así entender las exigencias que algunos agentes imponen en las organizaciones.

Rodríguez manifiesta que el mercado es el cúmulo de todos los compradores existentes y posibles de un producto o servicio, y que éstos tienen en común una necesidad o deseo específicos que se pueden satisfacer mediante intercambios [6]. Reisco por su parte, argumentan que el mercado es el organismo económico en el cual se produce el punto de coincidencia entre dos partes que intercambian un bien o servicio, y ese intercambio es un modo de aumentar la posesión de bienes y contratación servicios a disposición de las personas [7]. Ambas concepciones contemplan al mercado como el punto de encuentro para el intercambio de bienes materiales o servicios, cuya finalidad es sustentar las necesidades que presenta la sociedad; sin embargo, estas posturas imposibilitan considerar al mercado como un agente especializado, conocedor, analítico y determinado, que es capaz de condicionar a las organizaciones.

Para Nadal, el concepto de mercado es uno de los más trascendentales en la historia de las ciencias sociales, debido a la evolución que éste ha tenido y al profundo impacto que ha provocado en el sistema económico; para el autor, el mercado es el fundamento de la autonomía, y lo describe como un dispositivo social que se auto-regula y que mantiene la 
dimensión de lo económico, sin la intervención de los demás ámbitos de la vida social, pero ocasionando una fuerte repercusión sobre el contenido y los entornos de ellos [8]. Nadal no se equivoca al asegurar que el mercado es un mecanismo que tiene efecto en contextos como el social y el ambiental; no obstante lo anterior, su percepción queda limitada en la actualidad, al no apreciar que esa regulación que ejerce el mercado de manera autónoma, además de sostener la dimensión económica de la sociedad, también busca destacar áreas poco atendidas, que son la social y la ambiental; ¿Cómo pretende el mercado lograr esto? a través de una motivación afín a principios y valores relacionados con la recuperación del bienestar de la sociedad y el medio ambiente.

Kotler y Armstrong argumentan que el mercado es un grupo de posibles clientes que comparten una necesidad o deseo determinado y que podrían acceder a participar en una permuta de productos o servicios que los satisfaga [9]. Esta contribución se encuentra alejada de la concepción del presente artículo, debido a que éste último considera que el mercado juega un papel condicionante ante las organizaciones, y que además de mantener el interés de satisfacer una necesidad, manifiesta también la inclinación hacia la necesidad de una reestructuración equilibrada entre los atributos visibles de un producto, y las buenas prácticas que respaldan su producción.

$\mathrm{Si}$ bien hay distintitas connotaciones al respecto, esta investigación asume las precisiones hechas por Shepherd y Shepherd, quienes lo definen como un grupo de compradores y vendedores que comercializan bienes altamente intercambiables entre sí. De acuerdo a estos autores, delimitar un mercado y estudiar las condiciones que exige la demanda es extremadamente complejo, sin embargo, ambas pueden ser aproximadas considerando lo siguiente: a) elasticidad cruzada de la demanda, b) tipo de producto, c) dimensiones del producto y d) área geográfica; que para este caso en particular, se tomará en cuenta lo relacionado con el tipo de producto y el área geográfica [10]; la combinación de ambas consideraciones permite que un grupo de consumidores perfectamente localizados y especializados (área geográfica), exijan que un producto no solamente satisfaga sus necesidades más inmediatas, sino que éste sea capaza de avalar que los procedimientos sociales y ambientales que están detrás de su producción, son los correctos (tipo de producto).

En este sentido, es importante mencionar que el mercado no sólo exige los atributos que son implícitos al producto, sino que también requiere aquellos que se encuentran indirectamente relacionados a estos, pero directamente ligados a la organización y sus prácticas, es decir, los consumidores mantienen lo que se denomina "demandas intangibles". Las demandas intangibles de mercado son requerimientos que algunos agentes de mercado establecen de manera directa o indirecta a las organizaciones y que han propiciado cambios importantes en éstas [11], [12]. Respecto a esto último, Day aporta algo crucial por considerar, y es que en su investigación el autor asevera que el mercado representa a ese conjunto de consumidores agrupados que tiene la capacidad de condicionar a la organización [13]. Las demandas intangibles generalmente están asociadas a atributos no implícitos en el producto, es decir, están mayormente relacionadas al comportamiento de la organización, ya que buscan que éstas sean cada vez más sustentables.

Es en esta línea que Baumgartner contempla al mercado como uno de los componentes clave a considerarse para incorporar estrategias de sustentabilidad en las organizaciones; una de las estrategias que dicho autor discute es la relacionada con la apreciación de la sustentabilidad y la incorporación de sus principios a todos los procesos de la organización, cuya interiorización se percibe como una transición ética de sus dirigentes; la otra estrategia está ligada al hecho de que el principio ético de la sustentabilidad haya sido impuesto por exigencias de mercado [14]. El autor coincide con una parte crucial de este documento: el mercado impone a las organizaciones la implementación de estrategias relacionadas con la sustentabilidad para transitar a estructuras organizativas que satisfagan las nuevas necesidades de la sociedad.

De acuerdo a las investigaciones de Siebert y Martin, así como a las de Solís y colaboradores, el mercado no solo demanda atributos intrínsecos al producto, también exige que estos provengan de productores que practiquen y promuevan dos aspectos primordiales: 1) la responsabilidad social a favor de los trabajadores y 2) el cuidado ambiental; es decir, exigen atender aspectos que no se encuentran tangibles en el producto, lo que se traduce en demandas intangibles de mercado [15][16]. Los mercados se vuelven cada vez más dinámicos en su búsqueda de adaptación al modelo de desarrollo sustentable, con la finalidad de promover principios y valores que concuerden con tal esquema.

Estas últimas cuatro contribuciones evidencian el hecho de que existen consumidores especializados y localizados geográficamente que ejercen presión a las organizaciones para que éstas logren transitar hacia la sustentabilidad, demandando intangibilidades en el producto. Esta es la posición que concuerda con lo planteado en este escrito, misma que denota la situación en la que se encuentran actualmente las organizaciones.

Para fines del presente artículo, la demanda intangible de mercado se definirá como aquella exigencia de mercado que tiene que ver con aspectos no visibles en el producto final; relacionados éstos con la sustentabilidad social, ambiental y económica [14]-[16].

Esto implica la existencia de mercados que no solo demandan los atributos visibles en el producto, sino también que éstos provengan de productores que practiquen y promuevan dos aspectos primordiales: 1) la responsabilidad social a favor de los trabajadores [15]-[17] y 2) el cuidado ambiental [18]. En el primero de ellos se deben atender problemáticas como: trabajo infantil, incumplimiento en necesidades básicas de higiene y salubridad y salarios injustos [19]-[21]. En lo que respecta al segundo, los inconvenientes inmediatos que deben ser resueltos son: huella hídrica, huella de carbono e ineficiencia energética, entre otros [22]-[28], [18], [29]. La identificación de estas situaciones, así como la implementación de acciones para su resolución, representan un punto de partida en las organizaciones que procuran ir al encuentro de la sustentabilidad. 
Además de lo anterior, en la actualidad existe otra exigencia que se ha venido sumando a la lista de reclamaciones de mercado a ciertas organizaciones; éstas demandas tienen que ver con la equidad gerencial [30]-[31]. Dicha imposición ha sido externada por parte de los consumidores, quienes han considerado necesario incluir la participación igualitaria de las personas en la gerencia y demás posiciones de liderazgo; se argumenta que es benéfico para la organización, no sólo desde la perspectiva ética y moral, sino también desde la óptica de la eficiencia empresarial [32].

De esta manera, se puede apreciar la existencia de un segmento de consumidores preocupados por las prácticas de responsabilidad social y cuidado ambiental, como medio para coadyuvar a asegurar el bienestar de las futuras generaciones [33], y es en esta dimensión de mercado en donde actualmente se encuentran inmersas las organizaciones, donde la sustentabilidad se irgue como una condición de permanencia [34]. Este tipo de mercado es el que se ocupa en propiciar una conexión entre la organización y el desarrollo sustentable.

Los consumidores pretenden que las organizaciones avancen en la equidad gerencial y busquen hacer inclusión de personas sin importar su sexo, género, edad, religión, nacionalidad, preferencia política, etc., en los altos niveles jerárquicos [32]. Esto se ha convertido en la exigencia de un cambio para las organizaciones, debido a que se requiere una evolución en las estructuras organizativas que se manifieste en nuevas configuraciones organizacionales y nuevos gerentes [16]; principalmente porque existe la evidencia de que tan sólo el 5\% de mujeres se encuentran posicionadas en niveles de gerencia a nivel mundial [35], y solamente el $2 \%$ de personas con capacidades físicas diferentes ocupan una gerencia en México [36], situaciones que reflejan la falta de equidad en este nivel jerárquico [37], [38], [39], y que impiden que las organizaciones transiten hacia la sustentabilidad y se conviertan en organizaciones sustentables.

\section{B. Equidad gerencial}

El concepto de equidad gerencial no ha sido abordado ampliamente, sin embargo, para fines de este artículo y con el propósito de poder establecer un concepto claro, se debe explicar primeramente lo que es la "equidad", para después discutir lo relacionado con la "gerencia".

El concepto de equidad se cimenta en tres valores sociales: igualdad, acatamiento de derechos y justicia; la aceptación relativamente universal y la condescendencia hacia estos tres valores, permite que este concepto goce de aprobación [40]. Para este artículo, la equidad, a diferencia de la igualdad, considera que cada situación mantiene condiciones diferentes; sin embargo, se enfatiza en el hecho de que éstas no son impedimento para la imparcialidad. Para Rawls, la idea de equidad es primordial en el concepto de justicia, y hace hincapié en que los miembros de una sociedad deben debatir, cuestionar e instituir las prácticas que se definirán como justas, lo cual implica que las mismas dependerán de la disposición que exista entre los individuos pertenecientes a la colectividad en cuestión [41]. Este pensamiento acierta en considerar democráticamente la opinión de la colectividad para construir aquellas circunstancias que serán justas y ecuánimes, independientemente de las particularidades que predominan en los diferentes grupos de una comunidad.

Según Hernández, la equidad es un concepto comparativo entre grupos humanos que se realiza cuando se logra que los iguales sean tratados de la misma manera y que los desiguales sean tratados de una forma distinta o especial [42]; esta idea encaja perfectamente bien en grupos o comunidades que buscan el beneficio de un servicio público, como por ejemplo el de la salud; pero no coincide con lo planteado en este artículo, en donde las organizaciones pretenden valorar las desigualdades y aprovechar solamente aquellas capacidades que las pueden llevar a estructuras más amigables con el medio ambiente y la sociedad, claro, sin olvidar el propósito fundamental de crecer sus recursos económicos.

La Comisión Económica para América Latina y el Caribe (CEPAL) se refiere a la equidad como "un principio de ética y de justicia", y define la equidad como las mismas circunstancias y oportunidades para todas las personas sin excepción, solo haciendo ciertos ajustes en casos muy particulares, lo que permite de acuerdo a su percepción, avanzar hacia una sociedad más justa [43]; esta definición hace alusión, tan solo en la primera parte, a lo aquí propuesto: mismas condiciones y oportunidades para todos, sin ninguna consideración especial.

Con respecto al concepto de "gerencia", éste aparece como una traducción de la palabra en inglés "management", que también significa administración, gerencia, gobierno, dirección, gestión de entidades directivas, administración de recursos, dirección de empresas, etc. La gerencia tiene que ver principalmente con un procedimiento de planeación de actividades, recursos y resultados en las organizaciones a través de conocimientos, técnicas, métodos o herramientas para ser aplicados en procesos de producción, comercialización, mercadeo, colocación y expansión de las empresas.[44]. La gerencia permite que los subordinados cuenten con una guía superior que les apoye en el desempeño de sus actividades y el logro de un objetivo común.

Es por lo anterior que el concepto de "gerencia" ha sido considerado como un sistema holístico que permite escoger el agregado de empleados especializados y de alto conocimiento, que sean capaces de dirigir y resolver los asuntos de una organización [45]. Para Jean-Paul, la gerencia debe asumirse desde una perspectiva general, en donde se concretan propósitos dirigidos a adherir todas las fuerzas que definen la visión de una empresa, organización o institución, todo ello encaminado al logro de una mayor competitividad. Existen 3 conceptos clave en la gerencia: a) estrategia, con el fin de saber hacia dónde se dirige la compañía; b) organización, para poder llevar a cabo las estrategias; y c) cultura, con el objetivo de fortalecer la estructura organizativa [46]. Esta definición incluye un concepto importante, la competitividad; las organizaciones toman decisiones y acciones que buscan satisfacer las demandas de los consumidores, precisamente para posicionarse de una manera destacada en el mercado.

Ibáñez y Castillo argumentan que la gerencia es la ciencia social que se nutre del conjunto de conocimientos transdisciplinarios que se generan de la economía, psicología, ecología, biología, física, matemáticas, filosofía, religión, antropología, ingeniería, educación (entre otros), que se utilizan 
para actuar y tomar decisiones de impacto social basado tanto en la rigurosidad de su análisis, como en las reflexiones e interpretaciones que generan un entramado teórico-conceptual, que persigue el bienestar de las personas en un equilibrio con su entorno [48]. El presente autor ya contempla en su definición esa tregua que es tan necesaria entre la organización, los aspectos sociales y el entorno.

Hernández y Gómez afirman que la gerencia es la destreza y la sabiduría para trabajar con y a través de un equipo de subordinados, dirigiéndolos hacia el logro de los objetivos que plantea una organización. Esto implica construir en dicho equipo, las bases de conocimiento necesarias, pero de igual manera, requiere que el gerente se involucre con otras personas y corporaciones para lograrlo [48]. El edificar un equipo con los conocimientos precisos para desempeñar de manera correcta las funciones que requiere cada puesto en la organización, y la relación que debe establecer el gerente con los integrantes del mismo, así como con otras personas y organismos, entre ellos los consumidores, son puntos cruciales que aborda este artículo.

El término "gerencia" se enfoca en la disposición de los recursos con el propósito de generar beneficio económico y bienestar en los distintos componentes de la sociedad; el propósito crucial de la gerencia es prever, descubrir y revelar todo lo relacionado con la eficacia (logro de los objetivos), la eficiencia (logro de los objetivos con un óptimo uso de los recursos) y la efectividad social (impacto) en las organizaciones; además de lo anterior, la gerencia se ocupa también de los procesos de calidad, producción, marketing y competitividad [49]. El autor coincide en tres aspectos fundamentales que son contemplados en este trabajo: la gerencia es responsable de la generación de riqueza, el aprovechamiento de los recursos y el bienestar de la sociedad; es por esto que el profesionista que está a cargo de gerenciar, debe dejar de lado sus intereses personales, y debe actuar de acuerdo a las necesidades de la organización y sus trabajadores.

La gerencia obedece a una serie de funciones (cada una de ellas precisada) para ser ejecutadas de manera simultánea y continua, de acuerdo con cuatro aspectos primordiales: planteamiento, organización, dirección y control [50]. Es un proceso a través del cual se administran de manera óptima los recursos financieros, materiales y humanos para el logro de objetivos específicos, pero muy particularmente, para mantener satisfecho al cliente, que es lo que permite un buen posicionamiento de la organización en el mercado.

Sobre el concepto de equidad gerencial la literatura es relativamente escasa, sin embargo, Castro y Álvarez mencionan la equidad en la gerencia como una igualdad de oportunidades que se deriva de la interiorización de la responsabilidad social en las organizaciones y de la situación actual que se vive en los ámbitos social, cultural y científico. Las autoras argumentan que la equidad en la gerencia representa, entre otras cosas, la necesidad de asegurar la colaboración activa y ecuánime de todas las personas independientemente de su sexo, en todos los espacios y entornos de la acción corporativa e institucional, lo que conlleva a ocuparse en conseguir erradicar todo tipo de discriminación laboral [51]. Es entonces que el proceso de inclusión de la equidad como garantía de calidad y de excelencia empresarial, responde a la directriz que denota la evolución empresarial y a la necesidad de crear nuevos modelos organizacionales.

Otros abordajes del concepto de equidad en la gerencia se han realizado con el propósito de mejorar el desempeño de la organización, y se argumenta sobre la necesidad de concientizar a la sociedad y a quienes dirigen las organizaciones acerca de la importancia de originar en su interior políticas y medidas a través de las cuales se reconozcan los méritos, habilidades, competencias y otras características de las personas, por encima de su condición de sexo, raza, religión, edad, etc., a partir del fomento de ambientes laborales más diversos, tolerantes e inclusivos [52]. A medida que se va adquiriendo este mejoramiento en la estructura mental, laboral y cultural de quienes ocupan los niveles gerenciales en las organizaciones, se va obteniendo la capacidad de responder ante los cambios ocasionados por la globalización y los que han sido solicitados por el mercado.

Una aproximación al concepto de "equidad gerencial" la presenta Yates, quien la define como una solución a las dificultades y las inquietudes de la organización, y la oportunidad de una nueva orientación para unir esfuerzos ante la necesidad de una mayor equidad e inclusión para inhibir la discriminación laboral por raza, color, religión, edad, sexo u origen nacional. Hace hincapié en que este tipo de gerentes buscan activamente individuos que sean capaces y que merezcan oportunidades para ser preparados y asegurarse de que las barreras para su progreso serán mínimas [5].

Estos tres planteamientos son cruciales en el desarrollo del presente artículo; todos hacen alusión a la necesidad de incluir a las personas en las gerencias, independientemente de su condición, y considerando únicamente aquellas virtudes, cualidades y capacidades que contribuyan a la formación de trabajadores capaces y a la creación de ambientes laborales diversos; todo esto con el fin de alcanzar la excelencia organizacional.

Con base en estos argumentos, la información relacionada con equidad gerencial es escasa; y más escasas son aquellas investigaciones que tratan el tema de la equidad gerencial, como una demanda intangible de mercado que propicia cambios organizacionales para transitar hacia la sustentabilidad; es por eso crucial mencionar que hasta el momento de realizar esta revisión, las evidencias son limitadas.

Por tales razones y para fines del presente artículo, se construirá la definición del concepto de "equidad gerencial" a partir de las aportaciones de Castro y Álvarez, Vargas y Mota, y Yates, y se definirá como la unión de esfuerzos para inhibir la discriminación laboral [5], a través del reconocimiento de competencias y méritos de las personas [52] para su inclusión en una organización [51].

\section{ORGANIZACIÓN SUSTENTABLE}

El concepto de organización sustentable es el tercer concepto que fundamenta la conjetura propositiva del presente artículo. Sobre éste, se han presentado distintas posturas analíticas, sin embargo a continuación se discute el límite conceptual pertinente para el presente escrito, a partir de dos términos: 
"organización"y "desarrollo sustentable".

Sánchez define a la organización como la capacidad innata a la naturaleza de las personas, que se manifiesta en la coordinación de sus quehaceres en la vida de la sociedad; esta habilidad tiene para el autor la cualidad de aportarle a los individuos y grupos, adaptabilidad cuando se realizan modificaciones en el medio ambiente físico y social; se le puede considerar como esencial en el proceso de generación y acumulación de cultura, porque revela inteligencia para trascender en el tiempo y produce una forma de combinar a través de la interacción humana, ese talento individual, hasta convertirlo en estructuras sociales [53]. La organización parte de una necesidad muy básica del ser humano, la de "organizarse" en las distintas facetas que le ofrece su entorno; y él mismo es el que la lleva a evolucionar a una estructura compleja para lograr la adaptación a las distintas exigencias que surgen en los contextos económico, social y ambiental.

Otras posiciones señalan que la "organización" ha sido definida como un organismo social en el que el conjunto de sus integrantes desempeña un proceso sistematizado de actividades coordinado de forma consciente y racional, para la búsqueda de determinados objetivos; este conjunto de actividades y los fines de esta estructura, están influenciados por dos componentes: el entorno y las estrategias de los individuos [54]. De acuerdo con la percepción de las características y condiciones del entorno, se diseñan, construyen y ejecutan aquellas estrategias que sean más adecuadas para satisfacer las demandas de dicho entorno.

Desde una postura más pragmática, Drucker señala que la organización es un sistema social basado en la cooperación entre personas que requiere tres condiciones: dos o más personas interactuando, deseo de colaborar y un objetivo en común [55]. Aunque esta contribución es muy elemental, funge como punto de partida para otras aportaciones que han sido tomadas en cuenta para el desarrollo de este documento.

Andrade por su parte asegura que las organizaciones tienen un entorno complejo y que éste ha ocasionado que las organizaciones evolucionen a través del tiempo para afrontar los cambios; para el investigador las estructuras organizativas permiten el ajuste de las organizaciones a los cambios del ambiente, e indica que el contexto permite analizar un evento determinado [12]; en este sentido, las formas organizativas están directamente afectadas por dicho entorno, el cual se vuelve cada vez más competitivo, y que trae consigo amenazas, pero también oportunidades.

Robles y Garza en un capítulo del libro "Bienestar y desarrollo en el siglo XXI", presentan una precisión conceptual apropiada y la definen como un sistema social basado en la cooperación entre individuos en donde se requiere sean cumplidas cinco condiciones: 1) dos o más personas interactuando; 2) deseo de colaborar; 3) un objetivo en común; 4) un esfuerzo colectivo; y 5) un liderazgo ético que dirija y coordine el esfuerzo y los recursos de la organización [17]. Esta definición coincide con el compromiso que tienen los dirigentes de incorporar principios éticos y valores dentro de la organización.

En lo que respecta al significado de "desarrollo sustentable" las Naciones Unidas lo definen como: "aquel desarrollo que satisface las necesidades de las generaciones presentes sin comprometer la habilidad de futuras generaciones de satisfacer sus propias necesidades" [56]. Concepto que precisamente se deriva de una acción acordada entre naciones para promover un modelo de desarrollo económico mundial, que pueda ser compatible con la preservación del medio ambiente y la equidad social.

Calvente señala que el desarrollo sustentable es propiciar una interacción diferente entre la economía, el ambiente y la sociedad; asevera que en definitiva el deber del desarrollo sustentable no es frenar el progreso ni volver a estados arcaicos, sino lo opuesto, es promover necesariamente un progreso, pero desde un enfoque integral y más amplio [57]. Esto implica encontrar el equilibrio entre las necesidades y aspiraciones humanas, el mantenimiento de los sistemas naturales, los estilos de desarrollo, la forma de vida de las personas y las condiciones del entorno.

Morelli por su parte, afirma que la sustentabilidad en el desarrollo es una condición de equilibrio, armonía, resiliencia e interconexión que permite al ser humano satisfacer sus necesidades, sin exceder la capacidad de sus ecosistemas de apoyo para continuar restaurándose a sí mismos y proveyendo los servicios necesarios para satisfacer esas necesidades; de no incluirse la sustentabilidad en el desarrollo, el autor señala que se perderá la diversidad biológica y se eliminarán las fuentes de abastecimiento que son útiles para el sustento del individuo y la sociedad [58]. Esta definición da un poco más de realce a la parte ambiental de la sustentabilidad debido a que son los ecosistemas los que satisfacen las necesidades más básicas del ser humano; no obstante, este artículo mantiene interés por la triple hélice del desarrollo sustentable.

En esta misma línea, Velázquez y Vargas definen el desarrollo sustentable como el camino para encontrar el equilibrio económico, ecológico y social, dando como resultado la prosperidad y la captación de nuevos recursos [59]. Cualquier postura parcial que tome en consideración tan sólo una o dos de las tres dimensiones, limitará las iniciativas existentes para alcanzar el desarrollo sustentable. Reddy y Thompson recurren a una definición más básica del desarrollo sustentable, la cual coincide con la que se ha manejado por parte de las Naciones Unidas, que es "aquel desarrollo que permite satisfacer las necesidades del presente, sin comprometer la capacidad de las futuras generaciones para satisfacer las suyas" [60]. Esta definición ofrece puntos centrales de consenso que son imposibles de rechazar, pero podría ser más amplia en cuanto a procesos de trabajo específicos para facilitar la aplicación de estrategias del desarrollo sustentable y la obtención de resultados.

Los conceptos de organización y desarrollo sustentable mencionados en este último apartado, logran una fusión que permite dar una nueva orientación a la organización, lo que hace posible entender que la implementación de prácticas relacionadas con los tres ejes del desarrollo sustentable están positivamente relacionadas con una mejora de la imagen corporativa, evidenciada a través de distintos casos de estudio [61]. De acuerdo con el Instituto Internacional para el Desarrollo Sustentable, una organización sustentable es aquella 
que incorpora estrategias de negocios y actividades que satisfagan las necesidades de la organización y de los grupos de interés de éstas en el presente, mientras protege, sostiene e incrementa los recursos humanos y ambientales que serán necesitados por los siguientes grupos de interés en el futuro [62]. Esta definición va más allá al proponer el incremento de los recursos humanos y ambientales que serán necesitados por las futuras generaciones, lo que evidencia que aún existe y existirá una gran dependencia de ellos para el funcionamiento de cada una de las distintas actividades económicas que sostiene a la sociedad.

Dyllick y Hockerts las han definido como aquellas que satisfacen las necesidades de empleados, clientes, accionistas, comunidades, etc., sin comprometer su capacidad para satisfacer las necesidades de los futuros grupos de interés [63], aportación coincidente con el significado que otorgaron las Naciones Unidas al desarrollo sustentable.

Para Maio una organización sustentable es aquella que incluye procedimientos que se encuentran directamente relacionados con la preservación ecológica, el bienestar social y la responsabilidad financiera [64]. Por su parte Florea y colaboradores comentan que la organización sustentable es aquella que adquiere la capacidad de incorporar en sus operaciones las dimensiones: económica, social y ecológica. [65]. Ambos artículos, al igual que el presente, sostienen que la organización está sujeta a una evolución compleja debido a que se encuentra inmersa en un entorno económico, social y medioambiental que debe mantener con responsabilidad, ya que los tres la abastecen para generar riqueza.

En este sentido Hawken argumenta que la sustentabilidad se ha interiorizado en las corporaciones, y ha significado para ellas que el producto o servicio no debe competir en el mercado sólo en términos de imagen, poder, velocidad, empaque, etc. El autor en cuestión afirma que la compañía debe entregar productos o servicios al cliente de tal forma que se garantice el cuidado de los siguientes recursos: energía eléctrica, suelo y aire [66]. Actualmente hay otras estrategias que deben implementarse en la organización, y que están más bien relacionadas con la atención a aspectos sociales, por ejemplo, el trato digno y justo a los empleados.

Baumgartner y Ebner hacen énfasis en las tres dimensiones de la sustentabilidad que las organizaciones deben cumplir, la económica que está relacionada con el bienestar económico de las personas; la social que tiene que ver con el cuidado y la calidad de vida del trabajador, y que pretende influenciar de manera positiva las relaciones con sus grupos de interés en el presente y futuro; y finalmente la ambiental, que se refiere directamente a reducir las emisiones de sustancias tóxicas al aire, agua y suelo [67].

De acuerdo con estos autores, el significado de organización sustentable tiene su punto de partida en la definición del desarrollo sustentable del Reporte de Brundtland de 1987, que representa el principio ético de preocuparse y ocuparse en abatir la pobreza mientras se protege el medio ambiente en un nivel macro.

En consideración de los autores evaluados, se puede aseverar que este concepto puede ser incorporado en las organizaciones y que éstas, siguiendo la pauta del desarrollo sustentable, deben contener una interacción entre sus tres pilares, considerando en definitiva, sus impactos e interrelaciones [67]. Esta interiorización del desarrollo sustentable hace que las organizaciones tengan un progreso en sus estructuras y se preparen para resolver problemáticas más ininteligibles, por lo que tienen una influencia crucial en el desarrollo de sociedades sustentables.

De igual manera, Severo y coautores manifiestan que existen casos de estudio que demuestran que los conceptos de organización y desarrollo sustentable se pueden unir para dar una nueva orientación a la organización, en donde se puede entender que la implementación de prácticas de cuidado ambiental y responsabilidad social contribuye a un mejor desempeño organizacional [61]. La organización y el desarrollo sustentable en conjunto fortalecen los recursos y capacidades existentes para maximizar la obtención de beneficios desde una perspectiva tangible e intangible.

Por su parte, Solís y coautores definen el concepto de "organización sustentable" de la manera siguiente: aquella que ha integrado el desarrollo sustentable en sus tres grandes esferas: la económica, la social y la ambiental; en donde la interiorización de ese principio ético se estudia a través del mercado, ya que la organización sustentable trata de colocarse en una posición privilegiada entre sus consumidores, a través de la atención a sus exigencias en cuanto a los procesos que mantienen y que deben ser congruentes con las tres dimensiones en las que se fundamenta este principio [16]. El mercado exige que la organización cuente con una configuración sistémica ambientalmente apropiada, económicamente asequible y socialmente justa.

Para fines del presente proyecto investigativo, se construye la definición para "organización sustentable" como aquella que ha incorporado el principio ético del desarrollo sustentable en sus tres grandes componentes: el económico, el social y el ambiental [14] y [16].

\section{CONCLUSIONES}

El presente escrito permite señalar que la literatura existente relacionada con equidad gerencial es escasa, y es hasta ahora limitada la bibliografía que considera a la equidad gerencial, como una demanda intangible de mercado, que coadyuva a que surja el tránsito de las organizaciones hacia la sustentabilidad. Por ello, el presente artículo propone avanzar en esta línea mediante el postulado: "la equidad gerencial, como una demanda intangible de mercado, propicia cambios organizacionales hacia la sustentabilidad".

La construcción de dicho postulado es el punto de partida para la solución a las exigencias de mercado que obligan a las organizaciones a competir con atributos no intrínsecos al producto, tal como lo es el principio del desarrollo sustentable; y de igual manera, la construcción de esta hipótesis incorpora a la "equidad gerencial" en los estudios de mercado, lo que permite nuevas investigaciones en este rubro.

En trabajos futuros se planteará cómo la equidad gerencial, como una demanda intangible de mercado, se expresa en las 
exportadoras de uva de mesa sonorense, y cómo ésta coadyuva a la conformación de organizaciones sustentables.

\section{AGRADECIMIENTOS}

Los autores reconocemos el apoyo del Centro de Investigación en Alimentación y Desarrollo Regional (CIAD) de Hermosillo, Sonora, al igual que al Consejo Nacional de Ciencia y Tecnología (CONACyT), para poder realizar el presente proyecto de investigación.

\section{REFERENCIAS}

[1] Dorsch, M. J., Carlson, L., Raymond, M. A., y Ranson, R. "Customer equity management and strategic choices for sales managers", Journal of Personal Selling \& Sales Management, vol. 21, nº 2, pp. 157-166, 2001.

[2] Bruhn, M., Georgi, D., y Hadwic, K. "Customer equity management as formative second-order construct", Journal of Business Research, vol. $61 \mathrm{n}^{\circ} .12$, pp. 1292-1301, 2008.

[3] Cepeda, G., Ruiz, D. M., y Rondán, F. J. "Customer equity management in a professional player's agency: how much is worth my current player's base?", Revista de Psicología del Deporte, vol. 25, nº. 1, 2016.

[4] Hwang, J. H., Chung, J., Kim, J. W., Lee, D., y Yoo, W. S. “Antecedents to loyalty point redemption: Implications for customer equity management", Journal of Business Research, vol. 69, nº. 9, pp. 3731$3739,2016$.

[5] Yates, W. T. "Equity management: Affirmative action for the $21 \mathrm{st}$ century", Change: The Magazine of Higher Learning, vol. 25, $\mathrm{n}^{\circ} .2$, pp. 40-43, 1993

[6] Rodríguez, A. R. Fundamentos de Mercadotecnia: antología. Dirigida a estudiantes de mercadotecnia. Andalucía, España: Fundación Universitaria Andaluza, 2013 [En línea]. Disponible en: http://www.eumed.net/libros-gratis/2014/1364/\#indice.

[7] Resico, M. F. Introducción a la economía social de mercado. 1a ed. Buenos Aires: Konrad Adenauer Stiftung, 2010.

[8] Nadal, A. "El concepto de Mercado", UNAM: Conceptos y fenómenos fundamentales de nuestro tiempo, 2010 [En línea]. Disponible en: http://conceptos.sociales.unam.mx/conceptos_final/450trabajo.pdf.

[9] Kotler, P., y Armstrong, G. "Fundamentos de marketing", en Marketing en un mundo cambiante, cap 1. Ciudad de México: Pearson Educación, 2003, pp. 1-41.

[10] Shepherd, W., y Shepherd, J. The economics of industrial organization, Market Power's Effects on Prices, Profits, and Efficiency Cap 4. Illinois, EUA: Waveland Press, 2004, pp. 61-83.

[11] Aranda, A. N. "Liderazgo y Organizaciones Sustentables en el Sistema de Vid de Mesa Sonorense", Tesis de maestría dirigida por J. M. Robles, Departamento de Desarrollo Regional, Centro de Investigación en Alimentación y Desarrollo Regional (CIAD), Hermosillo, Sonora. Sep. 2016.

[12] Andrade, S. "Diseño y arquitectura organizativa en la globalización", Administración y organizaciones, vol. 15, pp. 71-92, 2005.

[13] Day, G. "Aligning the Organization with the market", MIT Sloan Management Review, vol. 48, $\mathrm{n}^{\circ} .1$, pp. 41-49, 2006.

[14] Baumgartner, R. "Organizational Culture and Leadership: Preconditions for the Development of a Sustainable Corporation", Sustainable Development, vol. 17, $\mathrm{n}^{\circ}$. 2, pp. 102-113, 2009.

[15] Siebert, S., y Martin, G. Managing people and organizations in changing contexts. London: Routledge, 2016.

[16] Solís, D. D., Robles, J. M., Preciado, J. M., y Hurtado, B. A. "El papel del mercado en la construcción de organizaciones sustentables", Estudios Sociales, vol. 27, nº. 49, pp. 274-294, 2017.

[17] Huesca, L., Camberos, M., y Calderón, C. "Bienestar y desarrollo en el siglo XXI", en Nuevas condicionantes en las organizaciones de los sistemas alimentarios: el caso del sistema Vid de Mesa de Sonora, cap. 10, J. M. Robles, y C. Garza, México: Plaza y Valdés Editores, 2011, pp. 191-206.

[18] Wei, J., Chen, Y., Tiemur, A., Wang, J., y Wu, B. "Degradation of pesticide residues by gaseous chlorine dioxide on table grapes", Postharvest Biology and Technology, vol. 137, pp. 142-148, 2018.

[19] Kresin, J. M. Analizing Value Added. The Packer. 2017 [En línea]. Disponible http://digitaledition.qwinc.com/publication/?i=396233\#\{\%22issue_id $\% 22: 396233, \% 22$ page $\% 22: 0\}$.

[20] Vanderhorst, D. "Argentina blueberry producers support prevention of child labor", The Packer, April 2018 [En linea]. Disponible en: https://www.thepacker.com/article/argentina-blueberry-producerssupport-prevention-child-labor.

[21] Karst, T. "California farm labor contractor to pay big penalty", The Packer, Feb. 2018 [En linea]. Disponible en: https://www.thepacker.com/article/california-farm-labor-contractorpay-big-penalty.

[22] Stefanelli, D., Goodwin, I., y Jones, R. "Minimal nitrogen and water use in horticulture: Effects on quality and content of selected nutrients", Food Research International, vol. 43, nº 7, pp. 1833-1843, 2010.

[23] Young, P. R., y Vivier, M. A. "Genetics and genomic approaches to improve grape quality for winemaking", Managing Wine Quality: Viticulture and Wine Quality, pp. 316-364, 2010.

[24] Kemp, L. J., Madsen, S. R., y Davis, J. "Women in business leadership: A comparative Study of countries in the Gulf Arab states", International Journal of Cross-Cultural Management, vol. 15, nº. 2, pp. 215-233, 2015.

[25] Conesa, M. R., Torres, R., Domingo, R., Navarro, H., Soto, F., y Pérez, A. "Maximum daily trunk shrinkage and stem water potential reference equations for irrigation scheduling in table grapes", Agricultural Water Management, vol. 172, pp. 51-61, 2016.

[26] Xiao, X., Li, Z., Matetic, M., Bakaric, M. B., y Zhang, X. "Energyefficient sensing method for table grapes cold chain management", Journal of Cleaner Production, vol. 152, pp. 77-87, 2017.

[27] Takma, D. K., y Korel, F. "Impact of preharvest and postharvest alginate treatments enriched with vanillin on postharvest decay, biochemical properties, quality and sensory attributes of table grapes", Food chemistry, vol. 221, pp. 187-195, 2017.

[28] Litskas, V. D., Irakleous, T., Tzortzakis, N., y Stavrinides, M. C. "Determining the carbon footprint of indigenous and introduced grape varieties through Life Cycle Assessment using the island of Cyprus as a case study", Journal of Cleaner Production, vol. 156, pp. 418-425, 2017.

[29] Ferrao, N., Mendonça, B. L., Marques, K., Ferreira, C., Canto, D., Flores, M. A., Galembeck, A., y Montenegro, Montenegro, L. T., y Montenegro, T. C. "Effects of fungal chitosan nanoparticles as ecofriendly edible coatings on the quality of postharvest table grapes", Postharvest Biology and Technology, vol. 139, pp. 56-66, 2018.

[30] Marrugo, L. "El acceso de la mujer a cargos de toma de decisiones en las empresas colombianas que cotizan en bolsa", Entramado, vol. 12, $\mathrm{n}^{\circ} .1$, pp. 108-120, 2016.

[31] Duque, Y. V., Ortiz, C., y Vargas, D. F., "Responsabilidad social y equidad de género: análisis de diez organizaciones adheridas al Pacto Global Colombia", Revista Tendencias \& Retos, vol. 18, nº. 1, pp. 111126, 2013

[32] Gabaldón, P. "Mujeres en los Consejos de Administración y en la Alta Dirección en España", Centro de Gobierno Corporativo, Investigaciones y Publicaciones, 2013 (I). [En línea]. Disponible en: https://directivasdearagon.es/wpcontent/uploads/2014/09/IE 2013 MUJERES opt.pdf

[33] The Packer, 2014. Fresh Trends. Estados Unidos: Vance Publishing Corporation. Grapes. [En línea]. Disponible en: http://www.thepacker.com/topics/grapes.

[34] Sroufe, R., y Gopalakrishna, V. "Management, Social Sustainability, Reputation, and Financial Performance Relationships: An Empirical Examination of US Firms", Organization \& Environment, vol. 32, issue. 3, pp. 331-362, Feb. 2018 [En linea]. Disponible en: https://journals.sagepub.com/doi/abs/10.1177/1086026618756611.

[35] Women's Major Group, "Igualdad de género, derechos y prioridades de las mujeres: Recomendaciones para los Objetivos de Desarrollo Sostenible (ODS) y la Agenda Post 2015", Biblioteca del Congreso de Chile/BCN, Sept. 2013. [En línea]. Disponible en: https://www.ben.cl/obtienearchivo?id=documentos/10221.1/43946/8/8 .pdf.

[36] Oficina Internacional del Trabajo (OIT) "Las mujeres en el trabajo: Tendencias de 2016", Ginebra, 2016. [En línea]. Disponible en: https://www.ilo.org/wcmsp5/groups/public/---dgreports/---dcomm/-publ/documents/publication/wcms_483214.pdf.

[37] Acker, J. "Inequality regimes: Gender, class, and race in organizations", Gender \& society, vol. 20, $\mathrm{n}^{\circ}$. 4, pp. 441-464, 2006.

[38] Alcaldía Mayor De Bogotá D. C. "Plan integral de acciones afirmativas, para el reconocimiento de la diversidad cultural y la garantía de los 
derechos de los y las afrodescendientes", Bogotá, 2012. [Enlínea]. Disponibleen:https://adsdatabase.ohchr.org/IssueLibrary/COLOMBIA Plan\%20Integral\%20de\%20Acciones\%20Afirmativas\%20para\%20el \%20Reconocimiento\%20de\%20la\%20Diversidad\%20Cultural\%20de $\% 2010$ \%20Afrodescendientes.pdf.

[39] Vela, E. "La discriminación en el empleo en México", Cap 2. México: Instituto Belisario Domínguez del Senado de la República, dic 2017.: https://www.conapred.org.mx/documentos_cedoc/completoDiscrimina cion08122017.pdf.

[40] Mokate, K. M. "Eficacia, eficiencia, equidad y sostenibilidad: ¿qué queremos decir?", Serie de Documentos de Trabajo I-24, BID, Banco Interamericano de Desarrollo, Washington D.C., jul, 2001 [En línea]. Disponible

en: https://publications.iadb.org/es/publicacion/14536/eficacia-eficienciaequidad-y-sostenibilidad-que-queremos-decir.

[41] Rawls, J. "Justicia como equidad", Revista española de control externo, vol. 5, no. 13, pp. 129-158, 2003.

[42] Hernández, M. "El concepto de equidad y el debate sobre lo justo en salud", Revista de salud Pública, vol. 10, pp. 72-82, 2008.

[43] Comisión Económica para América Latina y el Caribe (CEPAL) Equidad, desarrollo y ciudadanía. Visión Global Tomo I. Coordinado por J. A. Ocampo. Colombia: Ed. Alfaomega, 2000.

[44] Martínez, J. "Nueva gerencia pública: Análisis comparativo de la administración estatal en México", Convergencia, vol. 12, nº 39, pp. 13-49, 2005.

[45] Drucker, P. F. "The Ecological Vision: Reflections on the Human Condition, BOOK REVIEW", Economist, vol. 326, pp. 96-96, 1993.

[46] Sallenave, J. P. La gerencia integral: ¡no le tema a la competencia, témale a la incompetencia! Bogotá: Grupo Editorial Norma, 1995.

[47] Ibáñez, N., y Castillo, R. "Hacia una epistemología de la gerencia", Revista Orbis, vol. 16, pp. 54-78, 2010.

[48] Hernández, J., y Gómez, D. "Una aproximación al concepto de gerencia y administración aplicado a la disciplina de enfermería”, Escola Anna Nery Revista de Enfermagem, vol. 14, no. 3, pp. 625-32, 2010.

[49] Díaz, M., y Torrealba, R. "La construcción discursiva del concepto de gerencia", Revista ciencias de la educación, vol. 38, pp. 15-35, 2011.

[50] Reinos, A. M. "La acción gerencial en el contexto de los cambios organizacionales", Red de Investigación Educativa, vol. 5, n ${ }^{\circ} .2$, pp. 37 52, 2013.

[51] Castro, C., y Álvarez, B. "La Igualdad en la Responsabilidad Social de las Empresas", CONOC $>$ E, Ayuntamiento de Castellón de la Plana 2016.http://www.castello.es/web20/archivos/contenidos/61/D021_Igu aldad RSE.pdf.

[52] Vargas, J., y Mota, C. "Gerencia social para la equidad de género en las organizaciones", International Journal of Good Conciensus, vol. 8, pp. 130-47, 2013.

[53] Sánchez, C. Análisis Organizacional en el Sector Rural, Psicología para el Desarrollo Rural. Colegio de Postgraduados, Montecillo, Estado de México. ISBN: 968-839-245-6. Pag. 122. 1994.

[54] Zapata, G. J., Mirabal, A., y Canet, M. T. "El entorno de la organización: un estudio de sus tipologías y su vinculación con la percepción directiva y el diseño organizativo", Ciencia y Sociedad, vol. 40, $\mathrm{n}^{\circ} . \quad 4, \quad$ p. 785-822, dic. 2015. Disponible en: https://repositoriobiblioteca.intec.edu.do/handle/123456789/1624.

[55] Drucker, P. The essential Drucker: The best of sixty years of Peter Drucker's essential writings on management. USA: Collins Business essentials, 2001.

[56] World Commission on Environment and Development (WCED), United Nations. Our common future. (Oxford Paperbacks). Reino Unido: Oxford University Press, 1987.

[57] Calvente, A. M. "El concepto moderno de sustentabilidad". UAIS Sustentabilidad. Socioecología y desarrollo sustentable. Universidad Abierta Interamericana Centro de Altos Estudios Globales, UAIS-SDS100-002, jun, 2007 [En Línea]. Disponible en: http://www.sustentabilidad.uai.edu.ar/pdf/sde/uais-sds-100-002\%20$\% 20$ sustentabilidad.pdf.

[58] Morelli, J. "Environmental Sustainability: A Definition for Environmental Professionals", Journal of Environmental Sustainability, vol. 1, nº. 1, pp. 1-9, 2011.

[59] Velázquez, L. V., y Vargas, J. G. "La sustentabilidad como modelo de desarrollo responsable y competitivo". Ingeniería de Recursos Naturales y del Ambiente, vol. 11, pp. 97-107, 2012.

[60] Reddy, T. L., y Thompson, R. J. "Environmental, Social and Economic Sustainability: Implications for Actuarial Science", Actuarial Society of
South Africa's 2014 Convention, Cape Town International Convention Centre, Oct. 22-23, 2014. [En Línea]. Disponible en:

https://actuarialsociety.org.za/convention/convention2014/assets/pdf/p apers/2014\%20ASSA\%20Reddy\%20Thomson.pdf.

[61] Severo, E. A. Ferro, J. C., Henri, E. C., y Hermann, C. "Cleaner Production, Environmental sustainability and organizational performance: an empirical study in the Brazilian Metal-Mechanic industry", Journal of Cleaner Production, vol. 96, pp. 118-125, 2015.

[62] International Institute for Sustainable Development (IISD), Deloitte \& Touche and World Business Council for Sustainable Development (WBCSD). Business Strategy for Sustainable Development. Leadership Accountability for the '90s. Manitoba: IISD. 1992.

[63] Dyllick, T., y Hockerts, K. "Beyond the business case for corporate sustainability", Business Strategy and the Environment, vol. 11, pp. 130-141, 2002.

[64] Maio, E. "Managing Brand in the new stakeholder environment", Journal of Business Ethics, vol. 44, n. 2/3, pp. 235-246, 2003.

[65] Florea, L., Cheung, Y., y Herndon, N. "For all good reasons: role of values in organizational sustainability", Journal Business of Ethics, vol. 114, pp. 393-408, 2013.

[66] Hawken, P. The ecology of commerce: A declaration of sustainability. New York: Harper Collins, 1993.

[67] Baumgartner, R. J., y Ebner, D. "Corporate sustainability strategies: sustainability profiles and maturity levels", Sustainable Development, vol. $18, \mathrm{n}^{\circ} .2$, pp. 76-89, 2010.

Karla Terán Samaniego. Ingeniera Industrial y de Sistemas, 2002, de la Universidad de Sonora. Especialidad en Desarrollo Sustentable, 2014, de la Universidad de Sonora. Maestría en Sustentabilidad, 2017, de la Universidad de Sonora. Actualmente estudiante de Doctorado en Desarrollo Regional del Centro de Investigación en Alimentación y Desarrollo CIAD de Hermosillo, Sonora, México.

Martin Robles Parra. Se graduó en la Escuela de Economía de la Universidad de Sonora. Cuenta con una Maestría en Ciencias con especialidad en Metodología de la Ciencia por el Instituto Politécnico Nacional (IPN) y un Doctorado en Dirección de Organizaciones por la Universidad Popular Autónoma del Estado de Puebla. Cuenta con treinta años de experiencia en investigación y docencia en las líneas de Organización, mercado, liderazgo y cadenas productivas agroalimentarias. Es responsable de la línea metodológica de los programas de maestría y doctorado en desarrollo regional del Centro de Investigación en Alimentación y desarrollo, A.C y presidente del consejo académico de la Coordinación de Desarrollo Regional.

Juan Martin Preciado Rodríguez. Ingeniero Industrial y de Sistemas por la Universidad de Sonora, con una especialización en el Programa Iberoamericano de Formación en Minería de Datos. Doctorado en Ingeniería en el área de Computación por el Instituto de Ingeniería de la Universidad Autónoma de Baja California. Actualmente adscrito a la Coordinación de Desarrollo Regional del CIAD, donde es responsable de los cursos de Métodos y Técnicas de Análisis de Datos I y II, impartidos en los programas de Maestría y de Doctorado en Desarrollo Regional. Las líneas de investigación, la aplicación y el desarrollo de modelos de extracción de conocimiento en bases de datos.

Diana Cristina López López. Tecnóloga en Química, 1997 e Ingeniera Industrial, 2009 de la Universidad Tecnológica de Pereira, Risaralda, Colombia. Magíster en Sistemas Integrados de Gestión de la Calidad de la Universidad Tecnológica de Pereira, Risaralda, Colombia, 2012. Estudiante de Doctorado en Desarrollo Regional del Centro de Investigación en Alimentación y Desarrollo CIAD de Hermosillo, Sonora, México. Actualmente docente tiempo completo del programa de Ingeniería Industrial en la Universidad Católica de Pereira UCP, Pereira, Risaralda, Colombia. 